\title{
Effect of Fibre Supplementation on Body Weight and Composition, Frequency of Eating and Dietary Choice in Overweight Individuals
}

\author{
Vicky A. Solah ${ }^{1, *}$, Deborah A. Kerr ${ }^{1}$, Wendy J. Hunt ${ }^{1}$, Stuart K. Johnson ${ }^{1}$, Carol J. Boushey ${ }^{2}$, \\ Edward J. Delp ${ }^{3}$, Xingqiong Meng ${ }^{4}$, Roland J. Gahler ${ }^{5}$, Anthony P. James ${ }^{1,6}$, \\ Aqif S. Mukhtar ${ }^{1,7}$, Haelee K. Fenton ${ }^{1}$ and Simon Wood ${ }^{1,8,9}$ \\ 1 School of Public Health, Faculty of Health Sciences, Curtin University, Perth WA 6845, Australia; \\ d.kerr@curtin.edu.au (D.A.K.); w.hunt@curtin.edu.au (W.J.H.); s.johnson@curtin.edu.au (S.K.J.); \\ T.P.James@curtin.edu.au (A.P.J.); Aqif.Mukhtar@curtin.edu.au (A.S.M.); h.fenton@curtin.edu.au (H.K.F.); \\ simonwood@shaw.ca (S.W.) \\ 2 Epidemiology Program, University of Hawaii Cancer Center, Honolulu, HI 96813, USA; \\ cjboushey@cc.hawaii.edu \\ 3 Video and Image Processing Laboratory, School of Electrical and Computer Engineering, Purdue University, \\ West Lafayette, IN 47907, USA; ace@ecn.purdue.edu \\ 4 Flinders Centre for Innovation in Cancer, School of Medicine, Flinders University, Adelaide 5001, Australia; \\ rosie.meng@flinders.edu.au \\ 5 Factors Group R \& D, Burnaby, BC V3N4S9, Canada; rgahler@naturalfactors.com \\ 6 Curtin Health Innovation Research Institute, Faculty of Health Sciences, Curtin University, \\ Perth WA 6845, Australia \\ 7 Centre for Population Health Research, Faculty of Health Sciences, Curtin University, \\ Perth WA 6845, Australia \\ 8 InovoBiologic Inc., Calgary, AB Y2N4Y7, Canada \\ 9 Food, Nutrition and Health Program, University of British Columbia, Vancouver, BC V6T1Z4, Canada \\ * Correspondence: v.solah@curtin.edu.au; Tel.: +61-8-9266-2771; Fax: +61-8-9266-2958
}

Received: 12 December 2016; Accepted: 13 February 2017; Published: 16 February 2017

\begin{abstract}
Fibre supplementation can potentially reduce energy intake and contribute to weight loss. The mechanism may be reduced frequency of eating, resulting in reduced food consumption. The objective of this research was to determine the effectiveness of fibre supplementation with PolyGlycople $\mathrm{X}^{\circledR}\left(\mathrm{PGX} \mathrm{X}^{\circledR}\right)$, on body weight and composition, frequency of eating and dietary intake in 118 overweight adults. In a three-arm, parallel, blind, randomised controlled trial participants were randomised to one of three groups; $4.5 \mathrm{~g}$ PGX as softgels (PGXS), $5 \mathrm{~g}$ PGX granules (PGXG) or $5 \mathrm{~g}$ rice flour (RF) control. Prior to supplementation and at 12 weeks, participants captured before and after images of all food and beverages consumed within 4 days using a mobile food record app (mFR). The mFR images were analysed for food group serving sizes and number of eating occasions. In the PGXG group, per-protocol analysis showed there was a significant reduction in waist circumference $(2.5 \mathrm{~cm} ; p=0.003)$. Subgroup analysis showed that PGXG supplementation at the recommended dose resulted in a reduction in body weight $(-1.4 \pm 0.10 \mathrm{~kg}, p<0.01)$, body mass index (BMI) reduction $(-0.5 \pm 0.10, p<0.01)$, reduced number of eating occasions $(-1.4 \pm 1.2, p<0.01)$ and a reduced intake of grain food $(-1.52 \pm 1.84$ serves, $p=0.019)$. PGXG at the recommended dose resulted in a reduction in weight and BMI which was significantly greater than that for $\operatorname{RF}(p=0.001)$. These results demonstrate the potential benefits of PGX fibre in controlling frequency of eating and in weight loss.
\end{abstract}

Keywords: fibre; weight; waist circumference; frequency; eating; PolyGlycopleX ${ }^{\circledR}$ (PGX) 


\section{Introduction}

The burden of chronic disease in many countries is increasing concomitant with the percentage of the population with a high body mass index (BMI) [1]. Providing tools to improve the healthiness of the food environment and assisting people to make healthy food choices are important in the prevention of excessive weight gain [1].

Dietary pattern evidence has shown that higher dietary quality i.e. consumption of mainly whole grains, vegetables, fruit, nuts, legumes, seafood, plant protein and low-fat dairy leads to marked reductions in all-cause, cardiovascular disease and cancer mortality [2,3]. Similarly, a healthy dietary pattern which includes consumption of fruits, vegetables, grains, combined with a lower intake of sweets, red meat and processed meat, lowers the risk of developing colorectal cancer [4].

Evidence supports the role of dietary fibre in improving metabolic health. A higher intake of fibre is associated with increased satiety and reduced energy intake and therefore may be important in obesity management [5-11].

Total fibre is the sum of both dietary fibre (complete, intrinsic non-digestible plant carbohydrates, lignin) and functional fibres (non-digestible carbohydrates that have been isolated but still have beneficial physiological effects) [12]. What constitutes a 'beneficial physiological effect' and the level of evidence required for this to be substantiated remains unspecified [13]. Solubility or the ability of a fibre to dissolve in water will affect water holding capacity, viscosity and fermentability [11]. The properties of fibre that are associated with appetite, energy intake and body weight include solubility [11]. Solubility remains a common classification technique, although it has been suggested that fibres should be categorised according to functional properties such as viscosity and fermentability [14], since not all fibre is equal in delivering a beneficial physiological effect.

PolyGlycopleX (PGX) is a commercial functional fibre complex, manufactured by a proprietary process (EnviroSimplex ${ }^{\circledR}$ ) from three dietary fibres: konjac glucomannan, sodium alginate, and xanthan gum [9]. PGX is a soluble viscous non-starch polysaccharide complex that has been identified as contributing to improved satiety, lipidaemia and glycaemia $[8-10,15,16]$.

In the modern food environment both portion size and frequency of eating have increased in the population [17]. Mattes [17] has suggested that increased frequency of eating is a major factor in weight gain and is linked to the increased energy intake and rising BMI trends. In addition to frequency of eating, the types and amount of food and beverages consumed at an eating occasion may influence energy intake. Aljuraiban et al. [18] suggest that modifying eating behaviour through more frequent meals of low energy density and high nutrient quality may be an important approach to controlling obesity.

A challenge for understanding the contribution of dietary factors is our ability to measure diet. This is even more difficult in overweight and obese participants who are more prone to misreporting or underreporting their energy intake $[19,20]$. In addition, details such as time of eating is important in examining eating frequency and are difficult to accurately capture with paper-based methods. Advancement in technology has made available new image-based food recording systems such as those using a mobile food record app (mFR) [21-25]. Among the advantages of mFRs is the provision of real-time data capture which allows for the extraction of information from the images on the timing and location of the eating occasion, without relying on individuals to report these details [22,23]. Thus the mFR app allows for more accurate capture of time of eating while reducing the burden to the participant of reporting food consumption [23,24]. A unique aspect of this study was the use of the mFR to capture frequency of eating and dietary intake.

The objective of this research was to determine the effectiveness of diet supplementation with the viscous and gel-forming fibre, PolyGlycopleX (PGX), on body weight and composition and to determine if frequency of eating and diet can explain subsequent changes in 118 overweight adults. 


\section{Materials and Methods}

\subsection{Study Design}

A three-arm, parallel, blind, randomised control trial was conducted to determine whether PGX supplementation would promote weight loss and reduce waist circumference and BMI, change dietary patterns and reduce the frequency of eating or number of eating occasions. Participants (118 in total) were divided between three groups, $4.5 \mathrm{~g}$ PGX as softgels (PGXS), $5 \mathrm{~g}$ PGX granules (PGXG) and $5 \mathrm{~g}$ rice flour (RF) control, (Figure 1) and were assessed at baseline and 12 weeks. This trial was registered with the Australian New Zealand Clinical Trials Registry (reference ACTRN12614000701628).

The food images sent via the mFR were stored by a participant identification number (ID) only. No personal information was stored with the images. The research was conducted in accordance with the principles proposed by the Australian Association for Research in Education (AARE), the Australian Vice-Chancellor's Committee (AVCC) and the National Health and Medical Research Council (NHMRC). The study was conducted in accordance with the Declaration of Helsinki and ethics approval was granted by the Human Research Ethics Committee, Curtin University (reference HR170/2014).

\subsection{Study Participants}

Participants, aged 25-70 years and with BMI $25-35 \mathrm{~kg} / \mathrm{m}^{2}$ were recruited through advertisements on Curtin University radio, as well as email communication systems. Individuals that expressed interest were screened for eligibility by completing a screening questionnaire. Participants were excluded if they were: (a) pregnant; (b) unable to complete the 12-week study; (c) undertaking extreme forms of exercise or dieting; (d) unable to attend the study centre; or (e) had an allergy to any food ingredient used in the study; (f) had previous or current renal, liver or respiratory failure; (g) had previous gastric or weight-loss surgery; (h) had any malabsorption conditions or (i) had current or recent dietary fibre supplementation. The participant flow diagram (Figure 1) lists the reasons for exclusion. Once assessed as eligible, further details of the study were provided and informed consent obtained.

\subsection{Baseline Assessments}

Participants who met the selection criteria had height, weight and waist circumference anthropometric measurements taken at baseline. A portable stadiometer was used to measure height; weight was measured using a pre-calibrated digital scale and waist was measured as described by Norton and Olds [26]. To electronically record food consumption, iPods with mFR application installed were given to the participants, who were asked to keep a 4-day mFR at baseline and during the twelfth week of the study.

Before beginning their baseline image-based 4-day food record, all participants received a 30-minute interactive training session by a single researcher who conducted individual or small group training sessions using PowerPoint slides on how to connect the iPod to the Wi-Fi and how to use the mFR app. Training sessions were held at Curtin University in a room with Wi-Fi access. The inclusion of a fiducial marker (a checkerboard pattern of known shape, size and colour) in all food record images gave a known reference of dimension and markings to assist with food identification and portion size estimation [25]. During training, participants were able to practice taking before and after images using food models. Collected food record images were automatically uploaded from the iPod touch, when in Wi-Fi range. The iPods were coded with the participant ID ensuring each image was tagged with the participant ID, date and time of eating occasion. Participant ID was used to identify the images on the server. The server was accessible by researchers only via password.

Prior to the commencement of supplementation and during the twelfth week of the study, participants took before and after images of all foods and drinks (excluding water) consumed over four consecutive days. Food consumption during the entire study was ad libitum. 


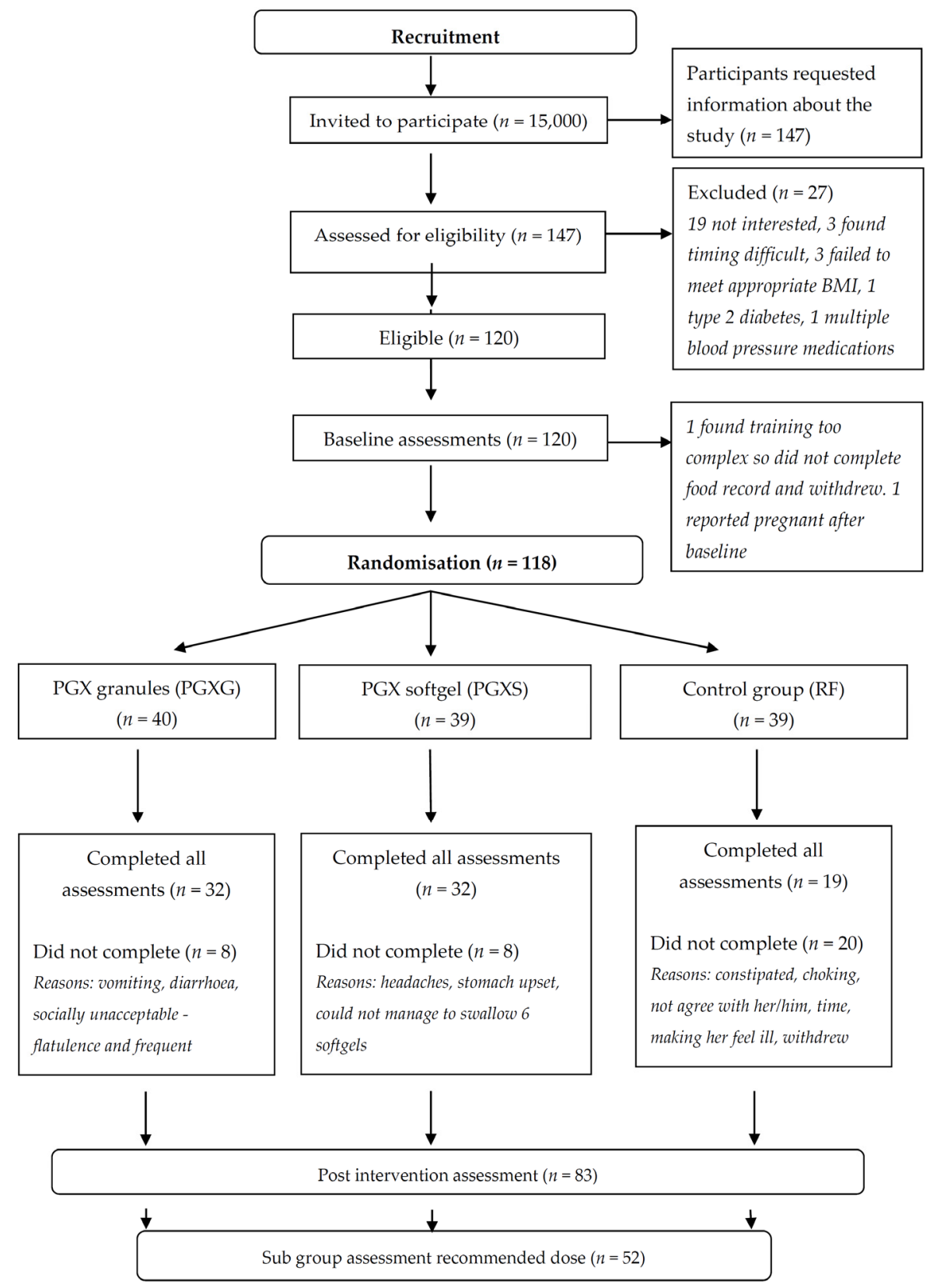

Figure 1. Participant flow diagram. RF: rice flour.

\subsection{PGX Supplementation}

The PGXS, PGXG and RF control supplements were provided to participants in a carry bag containing a 12-week supply labelled with a three-digit code. PGXG and the control were provided as $5 \mathrm{~g}$ individual doses in identical foil sachets and PGXS was provided in plain white jars each containing one month's supply. Research staff were blinded to the treatment allocation until all analyses were completed.

In Arm 1 (PGXS) instructions were provided in writing and verbally to the participants: "Take 1-2 softgels three times a day in week 1, 2-4 softgels three times a day in week 2 and 4-6 softgels, three times a day in week 3 to week 12". The recommended dose was four (4) to six (6) softgels containing $0.64 \mathrm{~g}$ fibre each, three times a day. This represented a supplement of dietary of between 7.6-11.4 g/day. Participants were also asked to consume $500 \mathrm{~mL}$ water with every softgel dose. Participants in Arm 2 were instructed to consume $5 \mathrm{~g}$ of PGXG containing $4.4 \mathrm{~g}$ fibre provided in a single dose foil 
sachet taken three times a day just before or with meals over the 12-week intervention period. This represented a supplement of dietary fibre of $12.2 \mathrm{~g}$ /day. Those in Arm 3 were provided with $5 \mathrm{~g}$ of RF containing $4 \mathrm{~g}$ fibre in the same dose format as the PGXG, representing $12 \mathrm{~g}$ fibre/day. RF was selected due to its neutral taste and hypoallergenicity, and it has a similar dietary fibre content, energy, colour and texture to PGX [7]. The recommended dose was 1 sachet, three times per day. Directions were "Stir 1 sachet into your meal or in a drink and consume immediately. You must consume 500mL of water each time you take a sachet." Participants were also advised that "If you have any discomfort, reduce the dose to 1 sachet a day for week 1, 2 sachets for week 2 and 3 sachets week 3 to week 12 . Contact the researcher to discuss any issues." Participants were informed of the importance of consuming water with the supplement as well as the possible gastrointestinal effects of fibre such as diarrhoea, bloating and flatulence as described in research by Kacinik et al. [7]. All participants were instructed to record their daily sachet or softgel intake and report in person to the researcher at the end of week 12 of the intervention.

\subsection{Post-Intervention Assessments}

At the end of the 12-week intervention period, measurement of participants' height, weight and waist circumference were repeated, along with the 4-day mFR being repeated during week 12 . At the end of the 12-week intervention, during the final meeting participants reported the number of doses to the interviewer and data were recorded in participant files.

\subsection{Dietary Analysis}

A researcher reviewed the 4-day food record images and as needed, confirmed the content of images with participants. Eating occasions were defined as all food and beverages (except water) and were taken from the image metadata and any additional notes supplied by the participant. Eating occasions were categorised as beverage only, food only, single item, food and beverage or fibre only. The images of fibre also allowed dose of fibre taken to be determined. Analysis was conducted to measure any change in eating occasions from baseline to study week 12 .

Images were analysed and eating occasions, types of foods and serving sizes were entered into a database specifically designed to capture the number of eating occasions and food groups according to the Australian Guide to Healthy Eating food group serves (vegetables, fruit, grain (cereal) foods, mostly wholegrain and/or high cereal fibre varieties, lean meats and poultry, fish, eggs, tofu, nuts and seeds and legumes/beans, milk, yoghurt cheese and/or alternatives, mostly reduced fat) (NHMRC 2013) plus junk food (Table 1).

The primary outcome variables measured at baseline and at the end of the intervention were: changes in weight, waist circumference and BMI; eating occasions and foods consumed each day classified as serves of junk food (energy-dense nutrient poor), grain (cereal), meat, dairy, fruits and vegetables for the three intervention groups. Within each intervention group, participants were further categorised into fibre dose compliance level and number of eating occasions and the primary outcomes for this subgroup were also evaluated.

\subsection{Statistical Analysis}

Per-protocol analyses were performed and reported. The distribution of all outcome variables, weight, waist and BMI were checked by construction of histograms to check normality. A mixed effect model with clustering of participants' ID and robust variance-covariance estimation were used to assess outcome variables. For per-protocol analysis, only those who completed the study at week 12 were included and missing values were not imputed. Further analysis of subgroups by actual dose consumed was performed and reported. All tests were two tailed and a $p$ value $<0.05$ was regarded as statistically significant. All analyses were performed using Stata MP 14.1 (Stata Corp., College Station, TX, USA). 
Table 1. Serve size in $\mathrm{g}$ or $\mathrm{mL}$ National Health and Medical Research Council (NHMRC), 2013 [27]. Fibre is 1 serve (g), with 2.5 serves daily minimum recommended dose.

\begin{tabular}{|c|c|c|c|c|c|c|c|c|c|c|c|c|c|c|}
\hline \multicolumn{4}{|c|}{ Grains } & \multirow{2}{*}{ Vegetable } & \multirow{2}{*}{ Fruit } & \multicolumn{3}{|c|}{ Meats } & \multicolumn{2}{|c|}{ Dairy } & \multicolumn{2}{|c|}{ Junk Food } & \multicolumn{2}{|c|}{ Fibre * } \\
\hline Bread & Porridge cooked & Muesli/Oats raw & Rice Pasta cooked & & & Beef Lamb Pork & Chicken & Fish & Milk & Cheese & Meat pie & Donut or Cake & PGXS & PGXG \\
\hline 40 & 120 & 30 & $75-120$ ( 1 / 2 cup $)$ & 75 & 150 & 65 & 80 & 100 & $250 \mathrm{~mL}$ & 40 & 60 & 40 & 3.8 & 4.4 \\
\hline
\end{tabular}

* Dose determined by study. PGXS = PGX softgel, $\mathrm{PGXG}=$ PGX granules. 


\section{Results}

\subsection{Baseline Characteristics}

\subsubsection{Per-Protocol Analysis}

Baseline measurements showing of all recruited participant characteristics according to treatment group are shown in Table 2. There were 92 females and 28 males, evenly distributed across the three treatment groups. In total 83 of the 118 participants (63\% retention) completed the 12-week study. Greater attrition was observed in the first two weeks of the supplementation for participants who reported stomach upsets and diarrhoea after PGXG consumption (attrition =6); who reported diarrhoea, headaches, difficulty swallowing recommended PGXS dose (attrition $=6$ ) and during the first six weeks of supplementation in the RF group (attrition =15) due to constipation and feeling ill.

Baseline data (Table 2$)$ shows the characteristics of study participants randomised $(n=118)$ and shows that at baseline there were no significant differences $(p>0.05)$ in age, height, weight, waist circumference, BMI and food group servings across three groups. At baseline PGXS participants had similar baseline frequency of eating (number of eating occasions) to the PGXG and RF participants but those allocated to the PGXG intervention had a significantly greater number of eating occasions per day than those allocated to the RF intervention $(p=0.04)$ (Table 2).

Table 2. Characteristics of all recruited study participants randomised at baseline $(n=120)$ comparing groups.

\begin{tabular}{|c|c|c|c|}
\hline All Participants & PGXS $(n=40)$ & PGXG $(n=40)$ & $\operatorname{RF}(n=40)$ \\
\hline Women & 31 & 30 & 31 \\
\hline Age (years) & $42.2 \pm 16.0$ & $46.5 \pm 14.0$ & $43.3 \pm 16.8$ \\
\hline Height $(\mathrm{cm})$ & $167.4 \pm 9.1$ & $167.3 \pm 9.0$ & $166.4 \pm 7.9$ \\
\hline Weight (kg) & $82.7 \pm 16.8$ & $80.9 \pm 16.6$ & $81.3 \pm 17.7$ \\
\hline Eating occasions per day & $5.4 \pm 2.8$ & $6.3 \pm 2.0$ & $4.8 \pm 2.1$ \\
\hline \multicolumn{4}{|l|}{ Food group servings (mean daily serves \pm SD) } \\
\hline Fruit $(150 \mathrm{~g})$ & $0.8 \pm 0.8$ & $1.0 \pm 1.0$ & $1.1 \pm 1.2$ \\
\hline Vegetable $(75 \mathrm{~g})$ & $2.4 \pm 1.5$ & $2.6 \pm 1.4$ & $2.5 \pm 1.1$ \\
\hline Grain (cereal) (40 g bread, 75-120 g cooked rice, pasta etc. or $500 \mathrm{~kJ}$ ) & $3.8 \pm 1.8$ & $4.3 \pm 2.2$ & $4.0 \pm 1.5$ \\
\hline
\end{tabular}

PGXS = PGX softgel, PGXG = PGX granules, RF = Rice Flour. SD = Standard Deviation.

\subsubsection{Subgroup Analysis}

Baseline measurements according to treatment group showing participant characteristics of all who consumed the recommended dose of fibre supplements are shown in Table 3. Baseline data (Table 3 ) shows the characteristics of study participants $(n=54)$ and shows that at baseline there were no significant differences $(p>0.05)$ in weight, waist circumference and BMI across three groups. PGXG and RF intervention sub-group numbers of eating occasions per day were not significantly different to each other or to the baseline group but the PGXS subgroup number of eating occasions was 7.4 times per day (Table 3). 
Table 3. Characteristics of study participants randomised at baseline $(n=54)$ comparing subgroups who consumed the recommended dose of fibre supplements.

\begin{tabular}{ccccc}
\hline All Participants & PGXS $(\boldsymbol{n}=\mathbf{1 7})$ & PGXG $(\boldsymbol{n}=\mathbf{1 8})$ & RF $(\boldsymbol{n}=\mathbf{1 7})$ & $\boldsymbol{p}$ Value \\
\hline Body weight $(\mathrm{kg})$ & $76.5 \pm 15.9$ & $87.7 \pm 20.2$ & $78.3 \pm 15.0$ & 0.62 \\
BMI $\left(\mathrm{kg} / \mathrm{m}^{2}\right)$ & $27.2 \pm 4.5$ & $28.7 \pm 5.2$ & $28.3 \pm 5.2$ & 0.64 \\
Waist $(\mathrm{cm})$ & $84.8 \pm 12.2$ & $89.2 \pm 20.4$ & $87.1 \pm 13.8$ & 0.59 \\
Eating occasions & $7.4 \pm 2.5$ & $6.0 \pm 2.0$ & $5.5 \pm 2.6$ & $p>0.05$ \\
per day & & & & \\
\hline
\end{tabular}

PGXS = PGX softgel, $\mathrm{PGXG}=$ PGX granules, $\mathrm{RF}=$ Rice Flour

\subsection{Effect of Intervention on Body Weight and Body Composition}

\subsubsection{Per-Protocol Analysis}

Per-protocol analysis revealed a significant reduction in waist circumference at week 12 of minus $2.5 \mathrm{~cm}$ (Confidence Interval $=-3.9,-0.8, p=0.003$ ) for those in the PGXG group (Table 4). No effect was seen on waist circumference for the PGXS and RF groups $(p>0.05)$. There was no effect of the PGXS or PGXG interventions on weight and BMI.

\subsubsection{Subgroup Analysis}

Analyses of the subgroup who consumed the recommended dose of fibre supplements showed a significant weight loss and reduction in BMI in the PGXG intervention compared to baseline (Table 5). However, the PGXS subgroup did not show a significant weight change during the intervention period. Compared to effect of the intervention on weight seen in the RF subgroup, that of the PGXG subgroup was significantly greater $(p=0.001)$.

Table 4. Change in participant characteristics from baseline to week 12 of the interventions of all participants who completed the interventions (per-protocol analysis).

\begin{tabular}{|c|c|c|c|}
\hline All Participants & PGXS $(n=32)$ & PGXG $(n=32)$ & RF $(n=19)$ \\
\hline Body weight (kg) & $0.47 \pm 1.85$ & $-0.49 \pm 0.34$ & $-0.03 \pm 0.58$ \\
\hline BMI $\left(\mathrm{kg} / \mathrm{m}^{2}\right)$ & $0.15 \pm 0.65$ & $-0.17 \pm 0.13$ & $0.01 \pm 0.20$ \\
\hline Waist $(\mathrm{cm})$ & $-0.17 \pm 2.92$ & $-2.50 \pm 0.60 p=0.03$ & $-1.3 \pm 1.0$ \\
\hline $\begin{array}{l}\text { Eating occasions per day } \\
\text { Food group servings } \\
\text { (mean daily serves } \pm \text { SD) }\end{array}$ & $-0.60 \pm 1.5$ & $-0.82 \pm 1.28 p=0.01$ & $-0.22 \pm 1.72$ \\
\hline Fruit $(150 \mathrm{~g})^{1}$ & $-0.2 \pm 0.76$ & $0.08 \pm 0.7$ & $-0.18 \pm 0.75$ \\
\hline Vegetable $(75 \mathrm{~g})^{1}$ & $-0.07 \pm 1.11$ & $-0.34 \pm 1.22$ & $-0.23 \pm 0.64$ \\
\hline Grain (cereal) & $0.21 \pm 1.73$ & $-0.79 \pm 1.66$ & $-0.51 \pm 1.23$ \\
\hline Dairy & $0.11 \pm 0.63$ & $-0.22 \pm 0.64$ & $0.07 \pm 0.35$ \\
\hline Junk food & $-0.14 \pm 2.00$ & $-0.57 \pm 1.29$ & $0.28 \pm 2.12$ \\
\hline Meat & $0.08 \pm 0.59$ & $0.01 \pm 0.79$ & $-0.09 \pm 0.78$ \\
\hline Alcohol & $-0.22 \pm 0.73$ & $0.17 \pm 0.99$ & $-0.02 \pm 0.26$ \\
\hline Fibre $(3.8-4.4 \mathrm{~g})^{1}$ & $1.89 \pm 0.91$ & $2.17 \pm 0.71$ & $2.35 \pm 0.58$ \\
\hline
\end{tabular}

Bold values denote significant within treatment effect. PGXS = PGX softgel, PGXG = PGX granules, RF $=$ Rice Flour.

${ }^{1} 1$ serve of fruit $=150 \mathrm{~g}, 1$ serve vegetable $=75 \mathrm{~g}, 1$ serve fibre $=3.8$ to $4.4 \mathrm{~g}$. 
Table 5. Change in participant characteristics from baseline to week 12 of the PGXS, PGXG and $\mathrm{RF}$ interventions in the subgroup analysis of those who consumed the recommended dose of fibre supplements.

\begin{tabular}{|c|c|c|c|}
\hline All Participants & PGXS $(n=17)$ & PGXG $(n=18)$ & RF $(n=17)$ \\
\hline Body weight (kg) & $0.22 \pm 1.61$ & $-1.4 \pm 0.10 p<0.01$ & $-0.03 \pm 0.58$ \\
\hline BMI $\left(\mathrm{kg} / \mathrm{m}^{2}\right)$ & $0.07 \pm 0.59$ & $-0.5 \pm 0.10 p<0.01$ & $0.01 \pm 0.20$ \\
\hline Waist $(\mathrm{cm})$ & $-1.04 \pm 2.28$ & $-1.2 \pm 1.00$ & $-1.3 \pm 1.0$ \\
\hline $\begin{array}{l}\text { Eating occasions per day } \\
\text { Food group servings } \\
\text { (mean daily serves } \pm \text { SD) }\end{array}$ & $-1.3 \pm 1.9$ & $-1.4 \pm 1.20 p<0.01$ & $-0.22 \pm 1.72$ \\
\hline Fruit $(150 \mathrm{~g})$ & $-0.43 \pm 0.59$ & $-0.63 \pm 0.57 p=0.022$ & $-0.18 \pm 0.75$ \\
\hline Vegetable $(75 \mathrm{~g})$ & $-0.35 \pm 0.96$ & $-0.82 \pm 1.31$ & $-0.23 \pm 0.64$ \\
\hline Grain (cereal) & $-0.93 \pm 1.47$ & $-1.52 \pm 1.84 p=0.019$ & $-0.51 \pm 1.23$ \\
\hline Dairy & $-0.05 \pm 0.56$ & $-0.59 \pm 0.50 p=0.012$ & $0.07 \pm 0.35$ \\
\hline Junk food & $-0.17 \pm 1.48$ & $-0.76 \pm 0.85$ & $0.28 \pm 2.12$ \\
\hline Meat & $-0.06 \pm 0.62$ & $0.18 \pm 0.90$ & $-0.09 \pm 0.78$ \\
\hline Alcohol & $-0.50 \pm 0.98$ & $0.11 \pm 0.32$ & $-0.02 \pm 0.26$ \\
\hline $\begin{array}{l}\text { Fibre supplement serves } \\
\text { (serving size } 4.5-5 \mathrm{~g} \text { ) }\end{array}$ & $2.6 \pm 0.47$ & $2.82 \pm 0.24$ & $2.35 \pm 0.58$ \\
\hline
\end{tabular}

Bold values denote significant within treatment effect. PGXS = PGX softgel, PGXG= PGX granules, RF = Rice Flour. $\mathrm{SD}=$ standard deviation .

\subsection{Number of Eating Occasions and Food Group Servings}

Collection of $\mathrm{mFR}$ images required participants to use the iPod provided by the study to record images before and after each eating occasion during the 4-day food record (Figure 2).

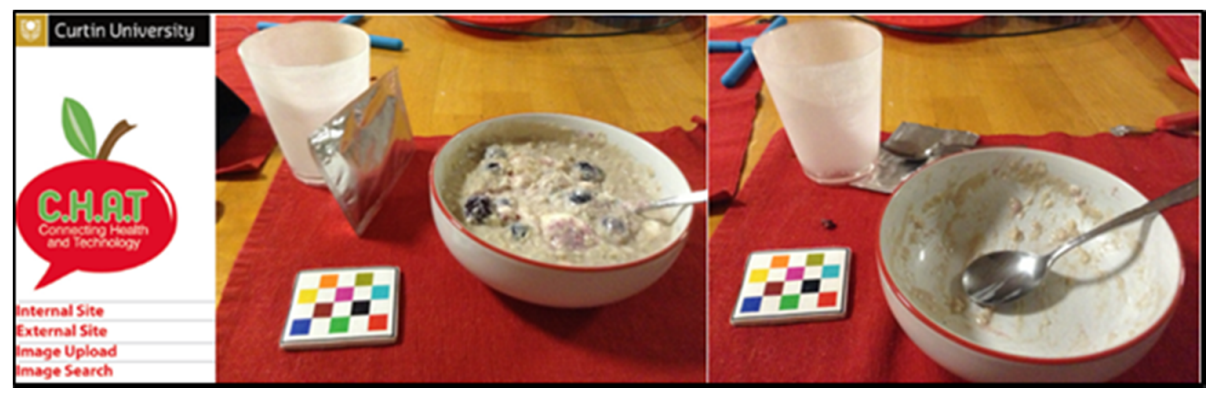

Figure 2. Before and after images of an eating occasion with foil sachet containing PGXG and fiducial marker captured with the mobile food record App on an iPod.

\subsubsection{Per-Protocol Analysis}

Analysis was conducted to measure change in number of eating occasions from baseline to week 12 of the interventions. The number of eating occasions was significantly reduced in the PGXG group $(p=0.01)$ during the intervention (Table 4$)$. No significant differences in number of eating occasions between baseline and week 12 were observed in the groups PGXS and RF or in food group servings in any of the intervention groups (Table 4) in per-protocol analyses.

\subsubsection{Subgroup Analysis}

For the subgroup analysis, the participants who consumed PGXG at the recommended dose of 2.5 to 3 times per day, significantly reduced their eating occasions by $1.4 \pm 1.2(\mathrm{CI}-2.1,-0.6, p<0.01)$ during the intervention period (Table 5).

Likewise, the participants who consumed PGXG at the recommended dose significantly reduced their daily intake of fruit $(-0.63 \pm 0.57$ serves, $p=0.02)$, dairy $(-0.59 \pm 0.50$ serves, $p=0.01)$ and grain food $(-1.52 \pm 1.84$ serves, $p=0.02)$ during the intervention (Table 5). Analysis of types of foods in 
the mFR showed that 1.6 to 2 serves of the grain food consumed at baseline were white bread for these participants. This consumption of white bread for the PGXG group also dropped significantly to 0.74 serves per day at 12 weeks of intervention.

\subsection{Adverse Events}

Adverse effects reported by the participants were mild and agreed with common reported reactions to increased fibre, for example those reported by Kacinik et al. (2011) [7] where for PGX supplementation, $30.9 \%$ of participants reported diarrhoea, bloating and flatulence whereas for rice flour consumption $7.8 \%$ reported constipation.

\section{Discussion}

This 12 week randomised controlled study showed that when consumed at the recommended dose (subgroup), the PGXG intervention gave a reduction in BMI and body weight, the number of eating occasions per day and consumption of servings of grain food.

The weight loss and BMI reduction observed for the PGXG intervention is most likely a result of compliance to the recommended dose, resulting in $64 \%$ of participants who consumed PGXG at the recommended dose losing weight and reducing BMI. The average 1.4-kg weight loss found in our study of overweight adults for the PGX per-protocol intervention is in agreement with previous research by Lyon et al. [28] who found a weight loss in women of $1.6 \mathrm{~kg}$ with 12 weeks PGX supplementation. Pal et al. [29] reported obese adults on 12-week supplementation of both artificially sweetened and flavoured PGX and psyllium lost weight (1.6 kg and $1.1 \mathrm{~kg}$ respectively).

On an individual basis, the highest weight loss in the PGXS group (per-protocol) was $4.4 \mathrm{~kg}$, with a waist circumference decrease of $5.5 \mathrm{~cm}$. However, in this group a weight gain of $6.3 \mathrm{~kg}$ and waist circumference increase was $9.6 \mathrm{~cm}$ was also recorded in one participant. This high variance in change in weight and waist circumference resulted in a non-significant weight loss for this group. Data collected by personal communication at the 6-week visit showed five participants in the PGXS group reporting that they expected PGXS to be a "magic bullet" for weight loss, which was an unexpected issue that requires consideration in future research. Protocol in future research may involve education of participants on using PGX to control appetite.

The significant reduction in waist circumference observed in the present study for PGXG per-protocol group of $2.5 \mathrm{~cm}$ was similar in magnitude to that reported in a study by Reimer et al. [8] where PGX consumption over 14 weeks resulted in a significant reduction of $1.96 \mathrm{~cm}$ in waist circumference. The significant reduction in BMI of 0.5 observed in the present study for PGXG sub-group was in contrast to Reimer [8] who found no significant differences in BMI compared to baseline.

The findings from our study that PGXS and PGXG consumption (per-protocol groups) did not affect weight is similar to research on PGX consumption by Kacinik et al. [7] who reported no significant differences in weight loss between PGX and the placebo groups in a study involving a low-calorie diet of $1000 \mathrm{kcal} /$ day for both treatments. There was also no weight loss recorded in a three-week study by Reimer et al. [30] where PGX was pre-mixed with breakfast cereal and consumed with yogurt. In our study participants were not on an energy restricted diet nor were the PGX and RF doses mixed with other products.

The use of the mFR allowed our research to determine level of compliance to the recommended dose of the PGXG subgroup by reviewing the images collected. The mFR image analysis of the foods eaten, serving sizes and number of eating occasions per day enabled important information about the dietary pattern of the overweight participants to be collected. The reduced number of eating occasions found with the PGXG subgroup, translated into reduced consumption of grain food of 1.52 serves $(p=0.019)$, mainly being a reduction in consumption of white bread.

Research by Kerr et al. [23] reported a reduction in consumption of energy-dense nutrient-poor (EDNP) foods as a result of tailored dietary feedback using the mFR. In the present study there was 
a non-significant reduction in junk food consumption of 0.57 in the PGXG per-protocol group and 0.76 serves in the PGXG subgroup. Pollard et al. [31] also found overweight people were more likely than those who were a healthy weight to decrease their intake grain food when trying to lose weight, supporting the reduced grain food trend found in this research. Small reductions in conscious or mindful energy intake can improve weight gain [32] and the choice to reduce grain food intake found in this research may have been the contributor to weight loss. Although we observed a reduction in daily intake of fruit and dairy, which is not desirable based on dietary guidelines [27], the magnitude of this change was minor.

Data from the mFR showed participants in all groups, other than the PGXG subgroup, appeared to make no changes to their eating pattern, as after 12 weeks of intervention, food group servings were not significantly different to baseline. This finding is supported by previous studies [32-34] where it was reported that humans tend to consume a consistent weight or volume of food from day to day. While participants reported feeling full in previous PGXS and PGXG research $[9,10,35,36]$, most participants in this study did not appear to use appetite to change their eating patterns, except in the PGXG subgroup who consumed the recommended dose, most likely resulting reduced appetite and reduced food intake.

Dietary feedback using the mFR indicated a reduction of 1 serve white bread, each day, which may have been a contributor to the reduction of $40 \mathrm{~g}$ in weight daily in the PGXG per-protocol group [27]. In a previous study, a reduction of $31.5 \mathrm{~g}$ carbohydrate per day was recorded at 12 weeks after PGX supplementation using a 3-day food and drink diary [29]. The 3-day food and drink diary does not provided details on type carbohydrates foods, whereas the $\mathrm{mFR}$ reports provided detail of types of foods and serving sizes. Dietary intake records collected using the mFR can reduce the bias found in auto-assessment [24].

In the present study, PGXG was taken with $500 \mathrm{~mL}$ water immediately before or with a meal. The PGXG was mixed in the water, in juice or mixed into the meal. The mechanism for weight loss in the PGXG sub-group in the present study may have been as a result of reducing dietary energy density of the meals with which it was consumed by increasing the fibre and water content of meals while maintaining the volume of food eaten $[37,38]$. Decreasing dietary energy density has been shown to be useful in long-term weight loss [39].

The detailed dietary feedback from the mFR enabled this research to determine weight loss was possible when PGXG at the recommended dose was consumed and the reduction in the number of eating occasions may be part of the mechanism for this effect. Previous research indicates that a possible mechanism behind the appetite and body weight reduction effects of PGX may be related to circulating gut hormones [30]. Reimer et al. [30] reported increased peptide YY, which can slow gastric emptying and decreased ghrelin (an appetite stimulant) on consumption of PGX. More work is required to confirm the physiological mechanisms controlling these effects of PGX.

The detailed dietary feedback from the mFR enabled this research to more accurately determine food group changes after 12 weeks. The reason the PGXS, and PGXG per-protocol groups did not reduce their weight may be because they did not reduce the number of eating occasions or change their daily intake of the foods and continued to consume the amount and type of food usually eaten. In addition, research by Polidori et al. [40] reports that weight loss leads to increased appetite, and appetite increases by approximately $100 \mathrm{kcal} /$ day per $\mathrm{kg}$ of lost weight. The reason fibre such as PGXG helps some individuals in the control of appetite and not others requires more research.

\section{Limitation}

Meals and consumption of test products were self-administered; the possibility of non-compliance could not be avoided. In the current study misreporting of intake may have occurred due to participants not taking images of all food and beverages consumed. In addition, the assessment of food group serves by a trained analyst may not be sensitive enough to detect changes in dietary intake. 


\section{Conclusions}

Supplementation with PGX at the recommended dose resulted in a reduction in body weight $(\mathrm{kg})$, BMI $\left(\mathrm{kg} / \mathrm{m}^{2}\right)$, reduced frequency of eating and reduced intake of white bread. The weight loss and BMI reduction from baseline to 12 weeks was significantly greater for PGXG at the recommended dose than for the RF treatment. Dietary assessment using the mFR provided detailed information enabling accurate analysis of the number of eating occasions and changes to food group servings per day. Further research on reducing the frequency eating of specific foods, such as junk food is warranted. These results demonstrate the potential benefits of PGX fibre in controlling frequency of eating and in weight loss.

Acknowledgments: PGX ${ }^{\circledR}$ and PolyGlycople $X^{\circledR}$ are registered trademarks of InovoBiologic Inc., Calgary, AB, Canada. Financial support for the submitted work was provided by Factors Group, Australia Pty Ltd. RJG owns the Factors Group of Companies, which retains an interest in PGX. SW receives consulting fees from InovoBiologic Inc.

Author Contributions: V.A.S., D.A.K., W.J.H., X.M., S.K.J. and S.W. designed and conducted the research; C.J.B., D.A.K. and E.J.D. developed the $\mathrm{mFR}$ part of this research; R.J.G. critically reviewed the study design; X.M., V.A.S., A.S.M. and D.A.K. analysed the data; V.A.S., D.A.K., W.J.K., S.K.J., C.J.B., E.J.D., X.M., A.P.J., A.S.M., H.K.F. and S.W. wrote the paper; all authors read and approved the final manuscript.

Conflicts of Interest: The authors declare no conflict of interest.

\section{References}

1. Swinburn, B.; Kraak, V.; Rutter, H.; Vandevijvere, S.; Lobstein, T.; Sacks, G.; Gomes, F.; Marsh, T.; Magnusson, R. Strengthening of accountability systems to create healthy food environments and reduce global obesity. Lancet 2015, 385, 2534-2545. [CrossRef]

2. $\quad$ Liese, A.D.; Krebs-Smith, S.M.; Subar, A.F.; George, S.M.; Harmon, B.E.; Neuhouser, M.L.; Boushey, C.J.; Schap, T.E.; Reedy, J. The Dietary Patterns Methods Project: Synthesis of findings across cohorts and relevance to dietary guidance. J. Nutr. 2015, 145, 393-402. [CrossRef] [PubMed]

3. Reedy, J.; Krebs-Smith, S.M.; Miller, P.E.; Liese, A.D.; Kahle, L.L.; Park, Y.; Subar, A.F. Higher diet quality is associated with decreased risk of all-cause, cardiovascular disease, and cancer mortality among older adults. J. Nutr. 2014, 144, 881-889. [CrossRef] [PubMed]

4. Tayyem, R.F.; Bawadi, H.A.; Shehadah, I.; Agraib, L.M.; AbuMweis, S.S.; Al-Jaberi, T.; Al-Nusairr, M.; Bani-Hani, K.E.; Heath, D.D. Dietary patterns and colorectal cancer. Clin. Nutr. 2016, in press. [CrossRef] [PubMed]

5. Delzenne, N.M.; Cani, P.D. A place for dietary fiber in the management of the metabolic syndrome. Curr. Opin. Clin. Nutr. Metab. Care 2005, 8, 636-640. [CrossRef] [PubMed]

6. Kristensen, M.; Jensen, M.G. Dietary fibres in the regulation of appetite and food intake. Importance of viscosity. Appetite 2011, 56, 65-70. [CrossRef] [PubMed]

7. Kacinik, V.; Lyon, M.; Purnama, M.; Reimer, R.A.; Gahler, R.; Green, T.J.; Wood, S. Effect of PGX, a novel functional fibre supplement, on subjective ratings of appetite in overweight and obese women consuming a 3-day structured, low-calorie diet. Nutr. Diabetes 2011, 1, 1-8. [CrossRef] [PubMed]

8. Reimer, R.A.; Yamaguchi, H.; Eller, L.K.; Lyon, M.R.; Gahler, R.J.; Kacinik, V.; Juneja, P.; Wood, S. Changes in visceral adiposity and serum cholesterol with a novel viscous polysaccharide in Japanese adults with abdominal obesity. Obesity 2013, 21, E379-E387. [PubMed]

9. Solah, V.; Brand-Miller, J.; Atkinson, F.; Gahler, R.; Kacinik, V.; Lyon, M.; Wood, S. Dose-response effect of a novel functional fibre, PolyGlycopleX, PGX, on satiety. Appetite 2014, 77, 72-76. [CrossRef] [PubMed]

10. Solah, V.A.; O'Mara-Wallace, B.; Meng, X.; Gahler, R.J.; Kerr, D.A.; James, A.P.; Fenton, H.K.; Johnson, S.K.; Wood, S. Consumption of the Soluble Dietary Fibre Complex PolyGlycopleX ${ }^{\circledR}$ Reduces Glycaemia and Increases Satiety of a Standard Meal Postprandially. Nutrients 2016, 8, 268. [CrossRef] [PubMed]

11. Wanders, A.J.; Van Den Borne, J.J.G.C.; De Graaf, C.; Hulshof, T.; Jonathan, M.C.; Kristensen, M.; Mars, M.; Schols, H.A.; Feskens, E.J.M. Effects of dietary fibre on subjective appetite, energy intake and body weight: A systematic review of randomized controlled trials. Obes. Rev. 2011, 12, 724-739. [CrossRef] [PubMed]

12. Slavin, J.; Green, H. Dietary fibre and satiety. Nutr. Bull. 2007, 32, 32-42. [CrossRef] 
13. Mann, J.I.; Cummings, J.H. Possible implications for health of the different definitions of dietary fibre. Nutr. Metab. Cardiovasc. Dis. 2009, 19, 226-229. [CrossRef] [PubMed]

14. Slavin, J.L. Dietary fibre and body weight. Nutrition 2005, 21, 411-418. [CrossRef] [PubMed]

15. Brand-Miller, J.C.; Atkinson, F.S.; Gahler, R.J.; Kacinik, V.; Lyon, M.R; Wood, S. Effects of PGX, a novel functional fibre, on acute and delayed postprandial glycaemia. Eur. J. Clin. Nutr. 2010, 64, 1488-1493. [CrossRef] [PubMed]

16. Brand-Miller, J.C.; Atkinson, F.S.; Gahler, R.J.; Kacinik, V.; Lyon, M.R.; Wood, S. Effects of added PGX ${ }^{\circledR}$, a novel functional fibre, on the glycaemic index of starchy foods. Br. J. Nutr. 2012, 108, 245-248. [CrossRef] [PubMed]

17. Mattes, R. Energy intake and obesity: Ingestive frequency outweighs portion size. Physiol. Behav. 2015, 134, 110-118. [CrossRef] [PubMed]

18. Aljuraiban, G.S.; Chan, Q.; Griep, L.M.O.; Brown, I.J.; Daviglus, M.L.; Stamler, J.; Van Horn, L.; Elliott, P.; Frost, G.S.; INTERMAP Research Group. The impact of eating frequency and time of intake on nutrient quality and Body Mass Index: The INTERMAP Study, a Population-Based Study. J. Acad. Nutr. Diet. 2015, 115, 528-536. [CrossRef] [PubMed]

19. Thompson, F.E.; Subar, A.F. Dietary Assessment Methodology. In Nutrition in the Prevention and Treatment of Disease, 3rd ed.; Coulston, A.M., Boushey, C.J., Ferruzzi, M.G., Eds.; Elsevier Academic Press: San Diego, CA, USA, 2013.

20. Meng, X.; Kerr, D.A.; Zhu, K.; Devine, A.; Solah, V.A.; Wright, J.; Binns, C.W.; Prince, R.L. Under-reporting of energy intake in elderly Australian women is associated with a higher body mass index. J. Nutr. Health Aging 2013, 17, 112-118. [CrossRef] [PubMed]

21. Bosch, M.; Zhu, F.; Khanna, N.; Boushey, C.J.; Delp, E.J. Combining global and local features for food identification in dietary assessment. IEEE Trans. Image Process. 2011, 2011, 1789-1792. [PubMed]

22. Harray, A.J.; Boushey, C.J.; Pollard, C.M.; Delp, E.J.; Ahmad, Z.; Dhaliwal, S.S.; Mukhtar, S.A.; Kerr, D.A. A novel dietary assessment method to measure a healthy and sustainable diet using the Mobile Food Record: Protocol and methodology. Nutrients 2015, 7, 5375-5395. [CrossRef] [PubMed]

23. Kerr, D.A.; Pollard, C.M.; Howat, P.; Delp, E.J.; Pickering, M.; Kerr, K.R.; Dhaliwal, S.S.; Pratt, I.S.; Wright, J.; Boushey, C.J. Connecting Health and Technology (CHAT): Protocol of a randomized controlled trial to improve nutrition behaviours using mobile devices and tailored text messaging in young adults. BMC Public Health 2012, 12, 477. [CrossRef] [PubMed]

24. Kerr, D.A.; Harray, A.J.; Pollard, C.M.; Dhaliwal, S.S.; Delp, E.J.; Howat, P.A.; Pickering, M.R.; Ahmad, Z.; Meng, X.; Pratt, I.S.; et al. The connecting health and technology study: A 6-month randomized controlled trial to improve nutrition behaviours using a mobile food record and text messaging support in young adults. Int. J. Behav. Nutr. Phys. Act. 2016, 13, 52. [CrossRef] [PubMed]

25. Zhu, F.; Bosch, M.; Khanna, N.; Boushey, C.J.; Delp, E.J. Multiple hypotheses image segmentation and classification with application to dietary assessment. IEEE J. Biomed. Health Inform. 2015, 19, 377-388. [CrossRef] [PubMed]

26. Norton, K.; Olds, T. Anthropometrica; University of New South Wales Press: Sydney, Australia, 2000.

27. National Health and Medical Research Council. Eat for Health. Australian Dietary Guidelines Summary. Canberra: Australian Government, Department of Health and Ageing. 2013. Available online: http://www.nhmrc.gov.au/_files_nhmrc/publications/attachments/n55a_australian_dietary_ guidelines_susumma_131014.pdf (accessed on 2 February 2015).

28. Lyon, M.; Wood, S.; Pelletier, X.; Donazzolo, Y.; Gahler, R.; Bellisle, F. Effects of a 3-month supplementation with a novel soluble highly viscous polysaccharide on anthropometry and blood lipids in nondieting overweight or obese adults. J. Hum. Nutr. Diet. 2011, 24, 351-359. [CrossRef] [PubMed]

29. Pal, S.; Ho, S.; Gahler, R.J.; Wood, S. Effect on body weight and composition in overweight/obese Australian adults over 12 months consumption of two different types of fibre supplementation in a randomized trial. Nutr. Metab. 2016, 13, 82. [CrossRef] [PubMed]

30. Reimer, R.A.; Pelletier, X.; Carabin, I.G.; Lyon, M.; Gahler, R.; Parnell, J.A.; Woods, S. Increased plasma PYY levels following supplementation with functional fiber PolyGlycopleX in healthy adults. Eur. J. Clin. Nutr. 2010, 64, 1186-1191. [CrossRef] [PubMed] 
31. Pollard, C.M.; Pulker, C.; Meng, X.; Denham, F.; Solah, V.; Scott, J.A.; Kerr, D.A. Cereal foods consumption trends and factors associated with changing intake, among Western Australian adults, 1995 to 2012. FASEB J. 2016, 30, 409.

32. Hill, J.O. Can a small-changes approach help address the obesity epidemic? A report of the Joint Task Force of the American Society for Nutrition, Institute of Food Technologists, and International Food Information Council. Am. J. Clin. Nutr. 2009, 89, 477-484. [CrossRef] [PubMed]

33. Bell, E.A.; Castellanos, V.H.; Pelkman, C.L.; Thorwart, M.L.; Rolls, B.J. Energy density of foods affects energy intake in normal-weight women. Am. J. Clin. Nutr. 1998, 67, 412-420.

34. Bell, E.A.; Rolls, B.J. Energy density of foods affects energy intake across multiple levels of fat content in lean and obese women. Am. J. Clin. Nutr. 2001, 73, 1010-1018. [PubMed]

35. Solah, V.A.; Meng, X.; Wood, S.; Gahler, R.J.; Kerr, D.A.; James, A.P.; Pal, S.; Fenton, H.K.; Johnson, S.K. Effect of training on the reliability of satiety evaluation and use of trained panellists to determine the satiety effect of dietary fibre: A randomised controlled trial. PLoS ONE 2015, 10, e012. [CrossRef] [PubMed]

36. Yong, M.K.; Solah, V.A.; Johnson, S.K.; Meng, X.; Kerr, D.A.; James, A.P.; Fenton, H.K.; Gahler, R.J.; Wood, S. Effects of a viscous-fibre supplemented evening meal and the following un-supplemented breakfast on post-prandial satiety responses in healthy women. Physiol. Behav. 2016, 154, 34-39. [CrossRef] [PubMed]

37. Rolls, B.J.; Castellanos, V.H.; Halford, J.C.; Kilara, A.; Panyam, D.; Pelkman, C.L.; Smith, G.P.; Thorwart, M.L. Volume of food consumed affects satiety in men. Am. J. Clin. Nutr. 1998, 67, 1170-1177. [PubMed]

38. Rolls, B.J. The relationship between dietary energy density and energy intake. Physiol. Behav. 2009, 97, 609-615. [CrossRef] [PubMed]

39. Ello-Martin, J.A.; Roe, L.S.; Ledikwe, J.H.; Beach, A.M.; Rolls, B.J. Dietary energy density in the treatment of obesity: A year-long trial comparing 2 weight-loss diets. Am. J. Clin. Nutr. 2007, 85, 1465-1477. [PubMed]

40. Polidori, D.; Sanghvi, A.; Seeley, R.J.; Hall, K.D. How strongly does appetite counter weight loss? Quantification of the feedback control of human energy intake. Obesity 2016, 24, 2289-2295. [CrossRef] [PubMed]

(C) 2017 by the authors; licensee MDPI, Basel, Switzerland. This article is an open access article distributed under the terms and conditions of the Creative Commons Attribution (CC BY) license (http:/ / creativecommons.org/licenses/by/4.0/). 